\title{
A NEW CHANNEL ASSIGNMENT METHOD IN COGNITIVE RADIO SYSTEM
}

\author{
C.P. Mallikarjuna Gowda ${ }^{1}$ and T. Vijayakumar ${ }^{2}$ \\ ${ }^{I}$ Department of Telecommunication Engineering, B.M.S. Institute of Technology and Management, India \\ ${ }^{2}$ Department of Electronics and Communication Engineering, SJB Institute of Technology, India
}

\begin{abstract}
In this paper, a novel method to estimate the unsuccessful probability is proposed by combining the unnecessary probability and the blocking probability. The Unnecessary probability is the probability when the mobile node is unnecessarily handed over and the blocking probability is the one when the network is not able to assign the channel to the mobile now. A new design of new channel assignment method for cognitive radio system is presented. The first fit method and the random assignment methods are also implemented for the cognitive radio application as a baseline. The PU and SU call blocking are modeled for all the three methods. It is shown that advantages of first fit method and the random assignment methods are combined into the new method, namely, selective assignment method. Simulation are conducted for the various network parameters like number of channels, number of links, load on each link, percentage of the PU versus $S U$ calls etc. It is shown that selective assignment method yields better results than that of the first fit method and the random assignment methods. Finally, the blocking probabilities are combined with the unnecessary handover probabilities of a three-node network to determine the unsuccessful handover probabilities of the first fit, random assignment and selective assignment methods.
\end{abstract}

Keywords:

Cognitive Radio, Random Assignment, First Fit Assignment, Selective Assignment, Unnecessary Probability, Blocking Probability, Unsuccessful Handover Probability

\section{INTRODUCTION}

Quality of service (QoS) is very important to ensure that the calls get transmitted from one point to other point. The QoS is not just necessary but it should be optimum enough to guarantee high throughput. The resources of the network should be effectively used with optimum QoS. The QoS can be optimized with the call arrival rate, call termination rate, holding time of the calls, blocking probability etc. In a cognitive radio world, there are licensed users and unlicensed users. The licensed users are known as Primary Users (PU) and the unlicensed users are known as Secondary Users (SU). In order to guarantee the interference free communication, the SU must not occupy the channels assigned for the PU. The Cognitive radio base station (CRBS) assigns the vacant channels to the PU and SU whenever the calls arrive.

The SUs are allocated the channels meant for the PUs. While allocating the SU in the channels of PU, it requires dynamically detecting and identifying the channels that are not in use by the PU. While performing this task of dynamic detection, there is a considerable amount of energy consumption. Ahmed et al. [1] and Elias et al. [2], authors discussed a comprehensive survey on the state-of-the-art channel assignment algorithms and spectrum assignment in cognitive radio networks, respectively. Usman et al. [3] [4] demonstrated channel assignment models for high priority users in wireless cellular network. The increase in blocking probability of newly originated calls is the tradeoff in using these models, which leads to bad QoS. Zafer et al. [5] developed a blocking probability analysis for a multi-hop wireless line and grid networks. The authors focused on the effect of transmission radius of the nodes and dynamic channel assignment algorithm. Xiukui et al. [6] demonstrated that the PU and SU can coexist in a network and the SU can directly access the channels allocated for the PU. The calls of the PU are closely monitored by the SU. A prediction technique has been proposed by the authors to guess the arrival of PU. However, this model lacks the channel updating scheme. The optimum architecture for the CRBS is discussed in detail by the authors in ref [7]. Bajpai et al. [8], authors discussed a spectrum allocation scheme for a Cooperative Cognitive Radio Network (CCRN). The scheme involved leasing out of the spectrum reserved for the PU to SU. However, this work does not cover the spectrum sensing methods.

Xiukui et al. [9] developed a prediction algorithm to predict the PU traffic and SU traffic. The prediction of the traffic was based on the spectrum sharing. The accessibility of the channels meant for the PU was estimated in terms of the probability for the SU allocation. The methods developed in this work are computationally expensive and it was difficult to implement this scheme practically. Authors of another similar research work [10] also performed analysis and developed a predictive algorithm to estimate the PU traffic. Cooperative spectrum sharing by means of static cognitive radio nodes over different service providers have been proposed by Kaniezhil et al. [11]. Centralized channel allocation was discussed in detail in [12]-[15]. A mobile switching center allocates the channels to the calls in centralized channel allocation since only mobile switching center has the information about the channel usage information. Mobile switching center allocates the channels in such way that there would be no interference of the channels. Whenever, each cell releases or allocates channels, it immediately notifies the mobile switching center and hence the later has all the information about the status of channels in all the cells. However, the centralized channel allocation system suffers a drawback. It suffers from the single point failure and the whole network system comes to halt when the mobile switching center fails. This scheme is not only scalable, but also not reliable.

The distributed channel allocation schemes [16]-[20] are more versatile and reliable than that of the centralized channel allocation schemes. There is no mobile switching center in the distributed channel allocation system and each cell has a base station to assign the channels. Each station takes the decision based on the local information and does not depend on the neighboring cells or base stations. However, the base station which allocates the channels, communicates with all other base stations about its decision so that the channel is not reserved or allocated by the other base stations.

The channel allocation algorithms by the base station can be based on the first fit or random assignment. In this work, the drawbacks of the first fit and random assignment are discussed 
and new selective assignment scheme is presented. The selective assignment method is designed to handle the calls of PU and SU.

Based on the literature review conducted, it is observed that the channel assignment methods are first fit and random assignment both for PU and SUs for same sequence of channels. When a new PU arrives, if the channel is already occupied by a $\mathrm{PU}$ or SU, then it results in blocking. Same thing happens for SU as well. Hence the blocking probabilities are high with first fit and random assignment. In order to overcome this, a new channel assignment that treats PUs and SUs separately is required to be designed.

Also, when a mobile node is transferred from one network to another network due to lack of enough bandwidth, if it results in unnecessary handover along with call blocking in the new network, then it is much more inefficient than being in the first network. Hence it is required to determine the total probabilities considering the unnecessary probability and blocking probability in a two or three network system, for example. An effort is made in this research work to determine the total unsuccessful probability considering both unnecessary handover probability and blocking probability for a three- network system. The literature for the methods to estimate the unnecessary handover can be found from [21]-[23].

In this paper, a new channel assignment method is proposed in order to reduce the blocking probabilities as low as possible. In the selective channel assignment, a window of channels can be reserved for the calls. Same window can be used both for PU and SU or separate windows can be reserved. When separate windows are reserved at two extreme ends of the channel sequence, the blocking probabilities are very least. When first fit or random assignment is used, the same channels will be allocated to the new PU or SU, but if they are occupied PU already then it results in blocking. In the new channel assignment method, the PU channels are reserved at one end of the sequence and the channels at the other end which are unused by PU, are reserved for the SUs. Hence the blocking probabilities are very less in such an arrangement. In this paper the selective channel assignment method is presented for different scenarios. Also, a method to estimate the total unsuccessful probability considering unnecessary handover probability and blocking probability is presented.

In section 2 , the probability equations derived and the channel selection methods first fit, random assignment and selective assignment methods are discussed in detail. Also, the probability equations are presented for the unnecessary handovers in a threenode network. In section 3, the simulation results for various cases are presented. Finally, the conclusions are given in the last section.

\section{PROBABILITY MODEL}

Let the Markov chain be represented as $M / M / \infty$. The arrival rate of the call is represented by $\lambda$ and termination rate by $\mu$. The states of the process are defined by no calls, one call, two calls, or $\mathrm{n}$ calls. As per the Markov condition, the probability of entering into a state depends only on the previous last state, but not on the previous state states of the last state. If the present state of the system is $P_{i}$, then the probability of transition is from $P_{i}$ to $P_{i+1}$. The state of any system is given by the Poisson distribution as:

$$
P(L, C)=\frac{L^{C}}{C !} e^{-L}
$$

where, $L$ is the load and the $C$ is the number of channels in the system. The Poisson's distribution is applicable for the number of calls being infinite, but practically, the number of channels is finite. If the $N$ is the number of infinite calls and $\mathrm{n}$ is the number of finite calls then by approximating the Poisson's distribution to finite number of calls, any call that is beyond n will not get service and assumed to be a NULL. Therefore, the Poisson's distribution now become,

$$
P(L, C)=\frac{\frac{L^{C}}{C !}}{\sum_{i=0}^{n} \frac{L^{i}}{i !}}
$$

where, $P_{e}(L, C)$ is known as the Erlangs probability density function. The blocking probability can be defined as:

$$
P_{b, p}=\frac{\frac{L^{n}}{n !}}{\sum_{i=0}^{n} \frac{L^{i}}{i !}}
$$

A network has the channels and the links. The number of links and channels are finite in a network. Each link may be considered as the connection between one base stations to another base station. Each base station can assign a call to a particular frequency range or channel and the call gets transmitted from one base station to the target end base station, to which the receiving call is connected to. In a cognitive radio networks, the type of calls can be primary or secondary. Hence each type of call can be through these channels and links. When the PU calls get the priority in transmission, SU calls also keep arriving at base station which must be given the secondary priority. Hence, when the PU calls are not scheduled, the SU are allocated in those channels and links. But as soon as the SU call is allocated, a PU call may arrive which leads to the blocking of the PU call.

Let the arrival rate of the PU call is $\lambda_{p}$ and that of the secondary call is $\lambda_{s}$ similarly $\mu_{p}$ and $\mu_{s}$ are the termination rates of PU and SU respectively. Let the states are represented by $x_{p}$ and $x_{s}$.

$$
\lambda_{p} P_{0 x s}=P_{1 x s} \mu_{p}
$$

This can be written as

$$
\begin{aligned}
& P_{1 x s}=\frac{\lambda_{p}}{\mu_{p}} P_{0 x s} \\
& P_{1 x s}=A_{p} P_{0 x s}
\end{aligned}
$$

Similarly, from second state to third state

$$
\begin{gathered}
\lambda_{p} P_{1 x s}=P_{2 x s} 2 \mu_{p} \\
P_{2 x s}=\frac{\lambda_{p}}{2 \mu_{p}} P_{0 x s} \\
P_{2 x s}=A_{p} P_{0 x s}
\end{gathered}
$$

This can also be written as

$$
P_{2 x s}=\frac{A_{p}^{2}}{2 !} P_{0 x s}
$$

The generalized form of Eq.(9) is 


$$
\begin{aligned}
& P_{x p, x s}=\frac{A_{p}^{x p}}{x p !} P_{0, x s} \\
& P_{x p, x s}=\frac{A_{s}^{x s}}{x s !} P_{x p, 0}
\end{aligned}
$$

Let $x_{s}=0$, then

$$
P_{x p, 0}=\frac{A_{p}^{x p}}{x p !} P_{0,0}
$$

Combining both Eq.(11) and Eq.(12),

$$
P_{x p, 0}=\frac{A_{p}^{x p}}{x p !} \frac{A_{s}^{x s}}{x s !} P_{0,0}
$$

Considering entire set of all the states,

$$
P_{x p, x s}=\frac{A_{p}^{x p}}{x p !} \frac{A_{s}^{x s}}{x s !} e^{\left[A_{p}+A_{s}\right]}
$$

The assignment of channels can be done in any of the two methods:

- First fit method

- Random assignment method

In First fit method, when a PU call or SU call arrives, the first link of first channel is assigned with that call. The call will move forwards in the chain of links. When the next call arrives, again the first link of the first channel is assigned. In this method, the main disadvantage is the channels at the farther end of the sequence of the channels are unutilized.

In the random assignment method, the channels are selected randomly; hence all the channels may get equal chance to service the calls. But there is a high chance that the calls get blocked since the calls are assigned randomly. There is a possibility that two consecutive calls may get assigned to the same channel. The advantage of using random assignment is all the channels are utilized uniformly.

In this paper, a new method is proposed, to assign both primary calls and secondary calls in a wireless network, the advantages of both the above methods are leveraged. This method is known as selective approach method. The channels can be assigned randomly but at the specific window of channels in the sequence. For examples, when the channels near the start of the sequence is selected in the window as priority channels, it becomes equivalent to First fit method. The window can be chosen randomly at any location; hence it has the advantage like a random assignment method. The location of the window is the key in this method to achieve the best performance. More importantly, the two windows can be chosen as priority for PU and SU separately. This particular feature is absent in the first fit and random assignment methods.

In some cases, the handover probabilities need to be considered along with the blocking probabilities for successful handover of the mobile unit. If the mobile units are handed over unnecessarily then if the calls get blocked, it results in a very severe situation and network will start losing its credibility. Hence before handing over, the unnecessary handovers are to be calculated so that risk can be estimated. In the present work, a three-node network simulated for the unnecessary handovers.
Consider there are three networks A, B and C as shown in Fig.1. For mathematical purpose, it can be denoted as nodes $n_{1}, n_{2}$ and $n_{3}$. Let the maximum number of channels in the network nodes are $B_{1}, B_{2}$ and $B_{3}$. Let the occupied bandwidth in the network nodes can be denoted as $b_{1}, b_{2}$ and $\mathrm{b}_{3} . P_{n j / n i}$ denotes the probability of mobile node moving from node $n_{i}$ to $n_{j}$ and $P_{n i / n i}$ denotes the probability of mobile node continue to stay in $n_{i}$ after a time interval $D$. The mobile node will be transferred from node $n_{1}$ to $n_{2}$ when $b_{2}-b_{1} \geq L$, where $L$ is the minimum gap of number of channels that should be maintained to take the decision for handover. More details can be found about wrong decision probabilities in [22].

Hence,

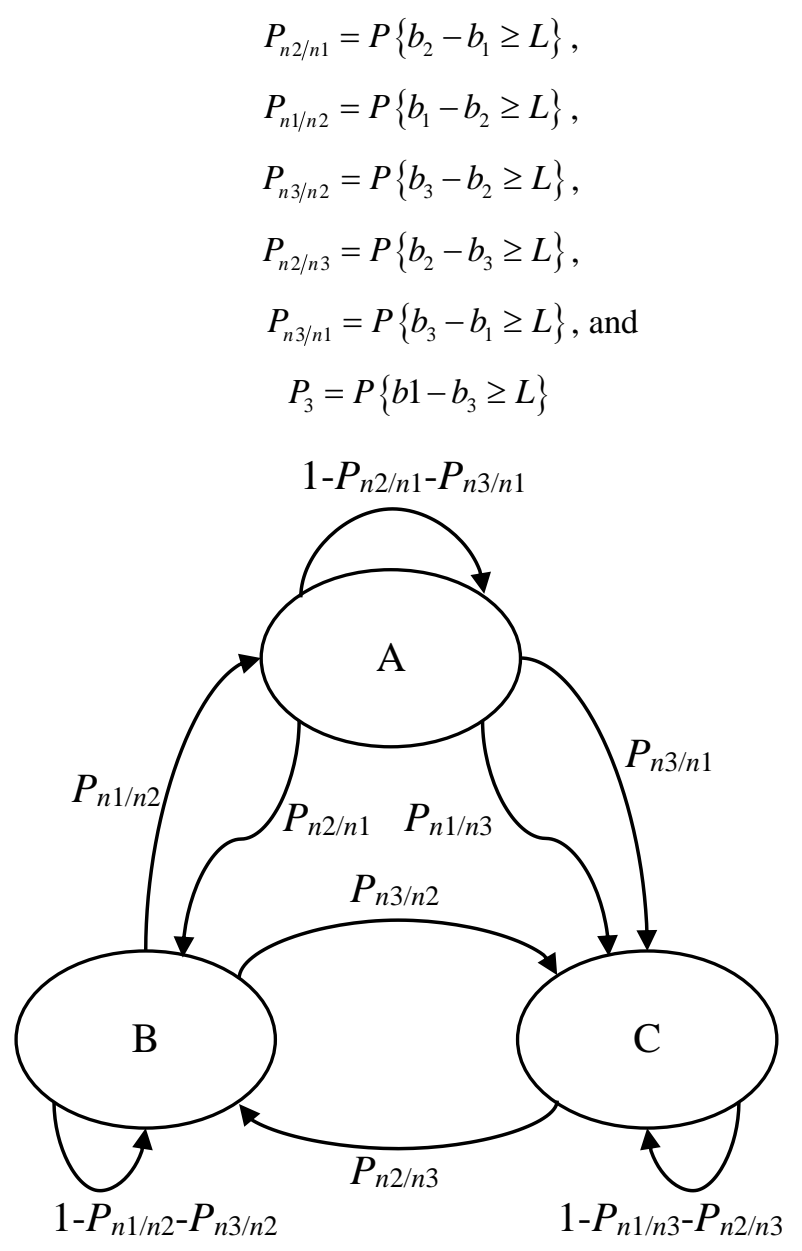

Fig.1. Three-Node Network

Unnecessary handover probability can be expressed as

$$
\begin{gathered}
U H P=P_{n 1} P_{n 2 / n 1} \sum_{j=L}^{B_{2}} \prod_{2, B_{2}-j} \sum_{k=0}^{j-L} \prod_{1, B_{1}-k} \xi_{1}(k, r, D) \cdot \sum_{i=0}^{B_{1}} \prod_{1, B_{1}-i} \sum_{k=i+L 2, B_{2}-k}^{B_{2}} \prod_{2} \psi_{2}(k, r, D) \\
+P_{n 1} P_{n 3 / n 1} \sum_{m=L 3, B_{3}-m}^{B_{3}} \sum_{k=0}^{m-L} \prod_{1, B_{1}-k} \xi_{1}(k, r, D) \cdot \sum_{i=0}^{B_{1}} \prod_{1, B_{1}-i} \sum_{k=i+L 3, B_{3}-k}^{B_{3}} \prod_{3}(k, r, D) \\
+P_{n 2} P_{n 1 / n 2} \sum_{j=L}^{B_{1}} \prod_{1, B_{1}-j} \sum_{k=j-L+2}^{B_{1}} \prod_{1, B_{1}-k} \psi_{1}(k, r, D) \cdot \sum_{i=0}^{B_{2}} \prod_{2, B_{2}-i} \sum_{k=0}^{i+L-2} \prod_{2, B_{2}-k} \xi_{2}(k, r, D)
\end{gathered}
$$




$$
\begin{aligned}
& +P_{n 2} P_{n 3 / n 2} \sum_{j=L 3, B_{3}-j}^{B_{3}} \sum_{k=j-L+23, B_{3}-k}^{B_{3}} \psi_{3}(k, r, D) \cdot \sum_{m=02, B_{2}-m}^{B_{2}} \prod_{k=0}^{m+L-2} \prod_{2, B_{2}-k} \xi_{2}(k, r, D) \\
& +P_{n 3} P_{n 1 / n 3} \sum_{m=L 1, B_{1}-m}^{B_{1}} \prod_{k=m-L+11, B_{1}-k}^{B_{1}} \prod_{1}(k, r, D) \cdot \sum_{i=0}^{B_{3}} \prod_{3, B_{3}-i} \sum_{k=0}^{i+L-2} \prod_{3, B_{3}-k} \xi_{3}(k, r, D) \\
& +P_{n 3} P_{n 2 / n 3} \sum_{j=L 2, B_{2}-j}^{B_{2}} \sum_{k=0}^{j-L} \prod_{3, B_{3}-k} \xi_{3}(k, r, D) \cdot \sum_{m=03, B_{3}-m}^{B_{3}} \sum_{k=m+L 2, B_{2}-k}^{B_{2}} \prod_{2}(k, r, D)(16)
\end{aligned}
$$

where, $\prod_{i, k}$ is the probability of occupied bandwidth $k$ and $\rho_{i}$ is the traffic load in channel $i$.

$$
\begin{gathered}
P_{n 1}=\frac{P_{n 1 / n 2}+P_{n 1 / n 3}}{P_{n 1 / n 2}+P_{n 2 / n 1}+P_{n 1 / n 3}+P_{n 3 / n 1}} \\
P_{n 2}=\frac{P_{n 2 / n 1}+P_{n 2 / n 3}}{P_{n 1 / n 2}+P_{n 2 / n 1}+P_{n 2 / n 3}+P_{n 3 / n 2}} \\
P_{n 3}=\frac{P_{n 3 / n 1}+P_{n 3 / n 2}}{P_{n 1 / n 3}+P_{n 3 / n 1}+P_{n 2 / n 3}+P_{n 3 / n 2}} \\
\prod_{i, k}=\frac{\rho_{i}^{k}}{k ! \sum_{j=0}^{B_{i}} \frac{\rho_{i}^{j}}{j !}} \\
\psi_{i}(r, k, D)=\sum_{m=0}^{B_{i}-k} \frac{\left(\Lambda_{i} D\right)^{m}}{m !} e^{-\Lambda_{i} D} \cdot\left[1-\sum_{s=0}^{m+k-r} \frac{\left(\lambda_{i} D\right)^{s}}{s !} e^{-\lambda_{i} D}\right] \\
\left.\sum_{i}=\lambda_{i}\left(B_{i}-k\right) / B_{i}-k\right)^{m} e^{-\Lambda_{i} D} \cdot \sum_{s=0}^{m+k-r} \frac{\left(\lambda_{i} D\right)^{s}}{s !} e^{-\lambda_{i} D}
\end{gathered}
$$

The unsuccessful handover probability can be expressed as

$$
P_{\text {unsuccessful }}=U H P \times P_{\text {blocking }}
$$

The blocking probabilities are estimated based on the type of channel allocation method, i.e. first fit, random assignment or selective assignment.

\section{SIMULATION RESULTS}

In this section, simulation results are presented for algorithms based on first fit, random assignment and selective assignment methods for primary unit's allocation and secondary unit's allocation. In the first part only two existing methods, namely, first fit, random assignment is verified for performance. The models are simulated for a tandem - networks that has the 10 nodes, 10 channels with 3 Erlangs of load per link and 20 nodes, 11 channels with 5 Erlangs of load per link. In both the cases, the number of PU calls are $80 \%$ of the total calls received. That means, for 2000 iterations, there are 2000 calls received, of which 1600 calls are PU calls and the remaining 400 calls are SU calls.

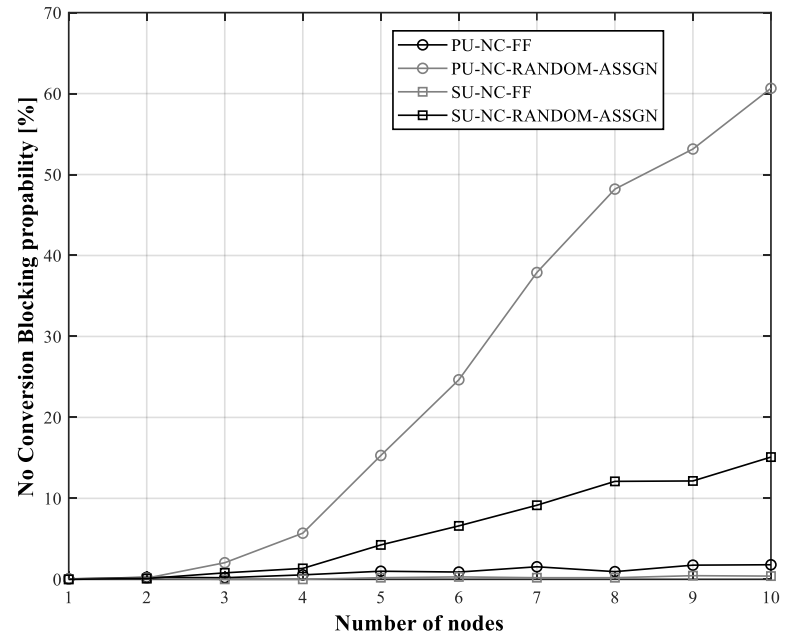

Fig.2. Blocking Probability of First Fit and Random Distribution for a load 3 Erlangs per link and with 10 Channels, 10 Links (nodes) and 2000 iterations

The Fig.2 shows the blocking probability of the PU and SU assignment with first fit and random assignments. The network has 10 Channels, 10 links and there was a load of 3 Erlangs per link. It can be concluded from Fig.2 that the random assignment has higher blocking probability than that of the first fit method both for the PU and SU. However, the problem with first fit method is, the channels in the tail end of the frequencies are not utilized to its capacity. There is always more utilization on the channels near the head end of the sequence of channels.

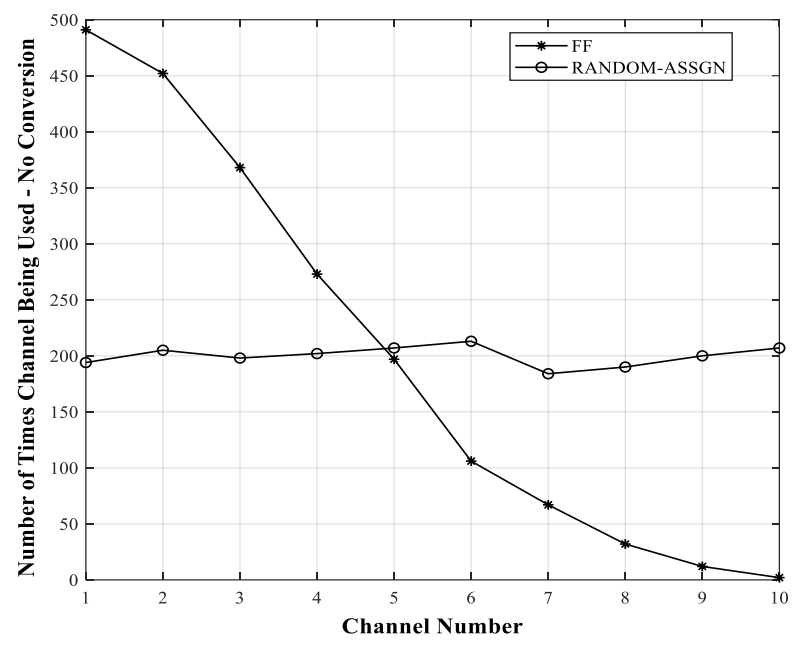

Fig.3. Frequency of channel usage by First Fit, Random Distribution for a load 3 Erlangs per link and with 10 Channels, 10 Links (nodes) and 2000 iterations

It can be observed from Fig.3 that with first fit method, channel 1 is used 485 times for assignment and Channel 2 is used 450 times, whereas the channels 7, 8, 9 and 10 are used less than 100 times. In case of the random assignment, all channels are used approximately 200 times, but the blocking probabilities are high as shown in Fig.2, i.e. $61 \%$ for PU and $16 \%$ for SU. In case for First fit, it is $3 \%$ and $1.5 \%$ respectively for PU and SU. 


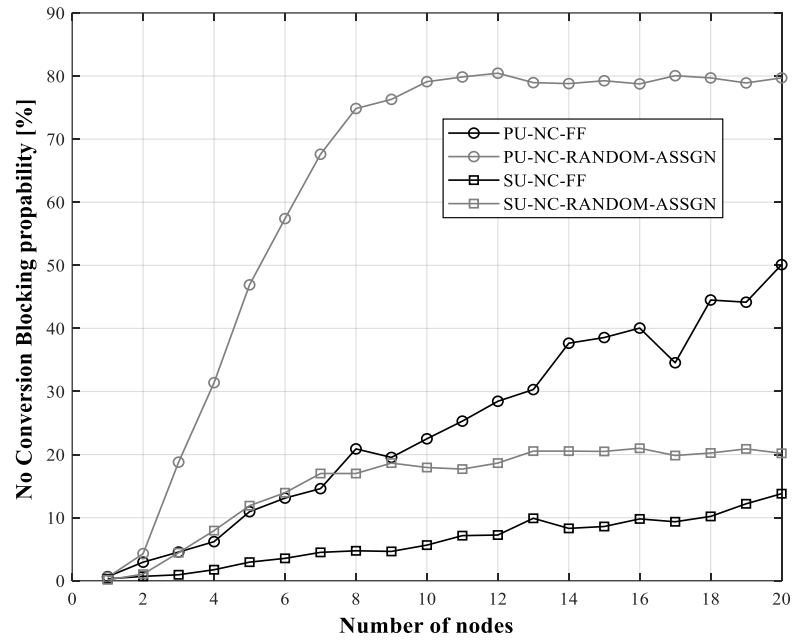

Fig.4. Blocking Probability of First Fit and Random Distribution for a load 5 Erlangs per link and with 20 Link (nodes), 11 Channels and 2000 iterations

When the load is increased to 5 Erlangs per link in a 20 links and 11 channel networks, the blocking probabilities are very high with $80 \%$ for PU and $20 \%$ for SU, as shown in Fig.4, when the random assignment was used. In case of first fit assignment, it is $50 \%$ for PU and $13 \%$ for SU. The increase in the blocking probabilities can be attributed to the high load on each link. The frequency distribution of channel assignment is provided in the Fig.5.

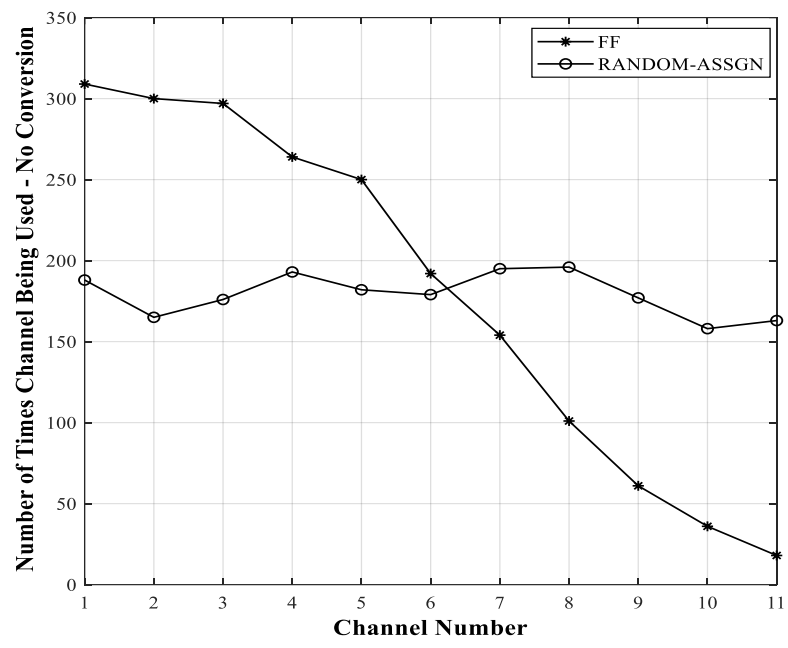

Fig.5. Frequency of channel usage by First Fit, Random Distribution for a load 5 Erlangs per link and with 20 Links (nodes), 11 Channels and 2000 iterations

As a next step, a selective assignment method has been developed as part of this research work. In this method, the channels are not selected based on the sequence, but based on the priority set. For example, the channels in the middle are given priority while assigning the calls or the channels at the end may be given the preference. This flexibility is not possible when the first fit method is used. The random assignment method does not have the flexibility to give the priority either at the beginning of the sequence or at any desired location. Hence the proposed selective assignment method has the advantage of the random assignment and the priority assignment similar to first fit method. Hence the selective assignment method may be treated as a combined method of first fit method and the random assignment method.

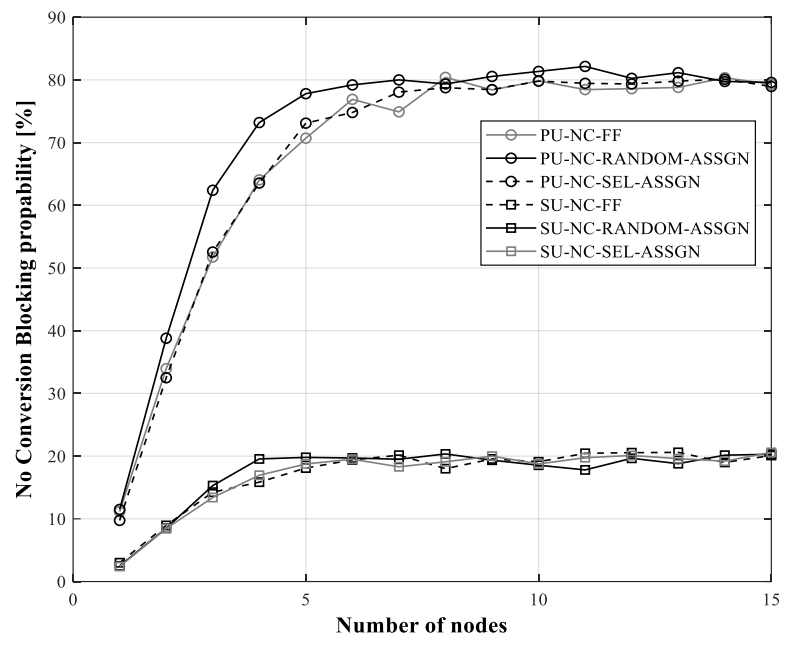

Fig.6. Blocking Probability of First Fit, Random Distribution and Selective Assignment methods for a load 8 Erlangs per link and with 15 Links (nodes), 10 Channels and 2000 iterations

The Fig.6 shows the blocking probability for the First Fit, Random Distribution and Selective Assignment methods with priority near the start of the channel sequence for a load 8 Erlangs per link and with 15 Links (nodes), 10 Channels and 2000 iterations. The simulations are performed both for PU and SU. Of the total 2000 calls, $80 \%$ of the calls are PU and the remaining $20 \%$ are SUs. It can be observed from the Fig.6 that selective assignment methods have better blocking probability than the first fit or random assignment. For example, at link 2, selective assignment has $32 \%$ blocking probability for $\mathrm{PU}$, random assignment has $39 \%$ and first fit has $34 \%$. After link 4, random assignment and selective assignment both have almost similar blocking probabilities. In case of SUs, the blocking probabilities are better in case of selective assignments up to the link 5. Since the number of SU calls are less than that of the PU calls, the blocking probabilities are much lower in case of SUs for all the three methods of assignment.

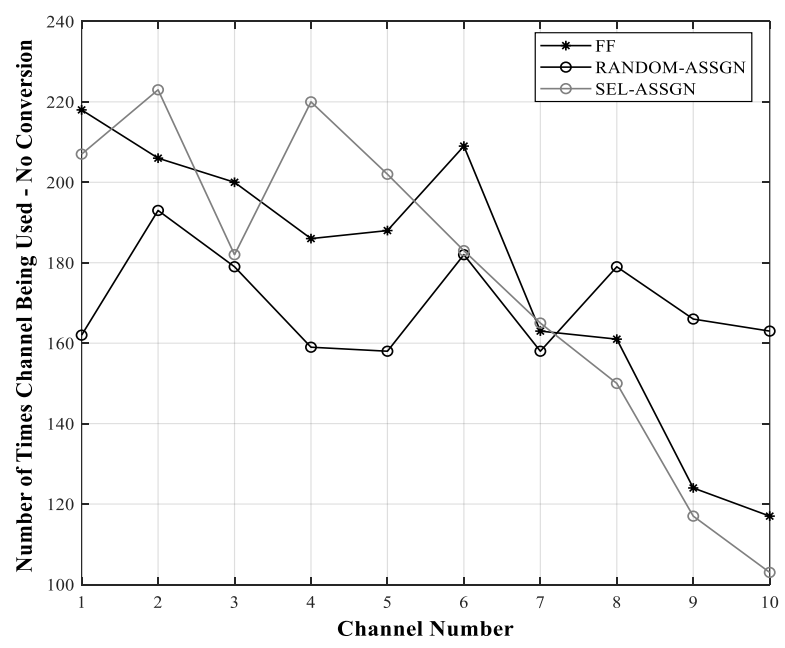

Fig.7. Frequency of channel usage by First Fit, Random Distribution and Selective Assignment methods for a load 8 Erlangs per link and with 15 Links (nodes), 10 Channels and 2000 iterations 
From Fig.7, it can be observed that the number of times channels near the start of the sequence is random up to channel 5 and then the frequency of channel usage is similar to first fit assignment. In both these cases, the cannels were not properly used.

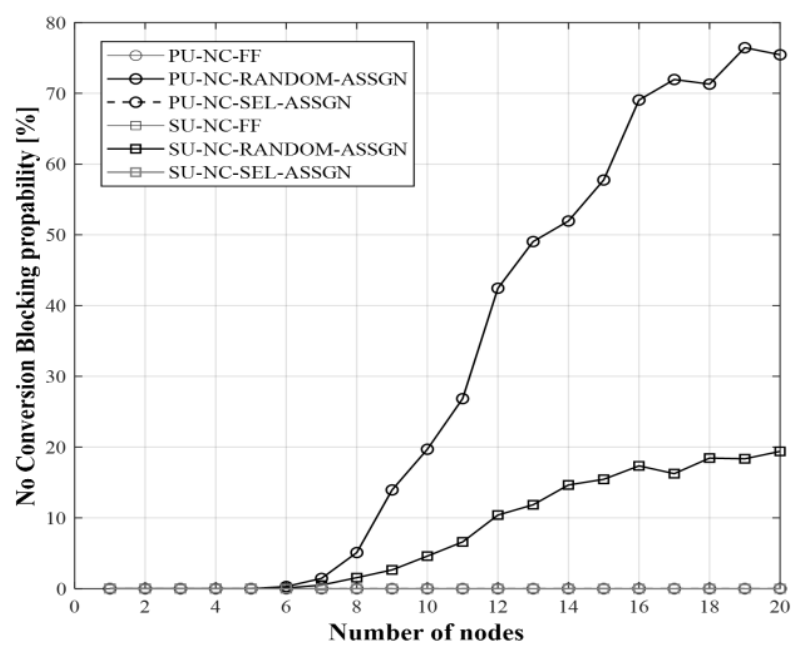

Fig.8. Blocking Probability of First Fit, Random Distribution and Selective Assignment methods for a load 15 Erlangs per link and with 20 Links (nodes), 50 Channels and 2000 iterations

The Fig.8 shows the blocking probability for the First Fit, Random Distribution and Selective Assignment methods with priority at the middle of the sequence of channels for a load 15 Erlangs per link and with 20 Links (nodes), 50 Channels and 2000 iterations. The Selective assignment and first fit methods both yielded almost zero blocking probability. This zero blocking probability was due to the reason that there are 50 channels available to accommodate 2000 calls. This is true both in cases of PU and SU.

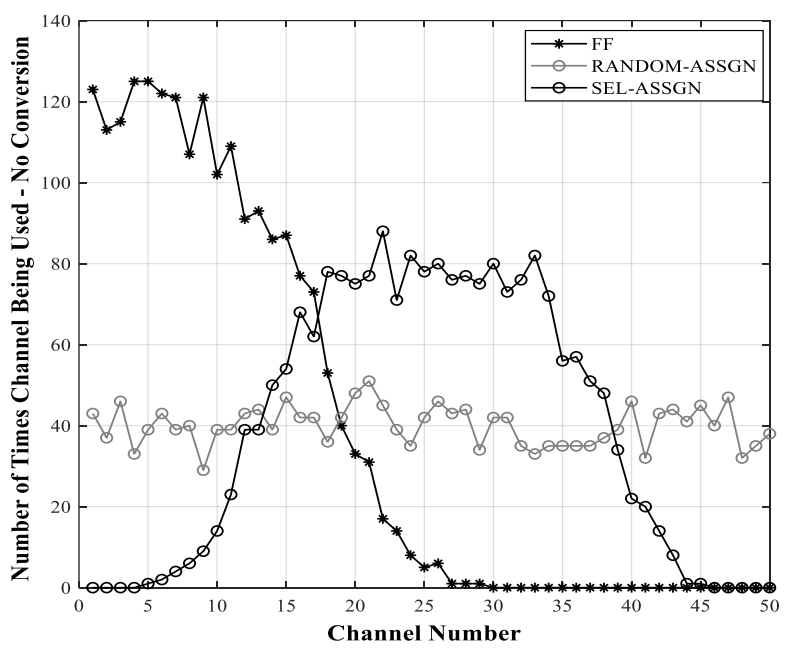

Fig.9. Frequency of channel usage by First Fit, Random Distribution and Selective Assignment methods for a load 15 Erlangs per link and with 20 Links (nodes), 50 Channels and 2000 iterations

The Fig.9 shows an interesting plot about the distribution of the utilization of the channels by the selective assignment. Since the method has the flexibility to provide priority to any window, in this simulation, the channels at the middle of the sequence were given priority both for PU and SU. The frequency of utilization is high near channel 25.

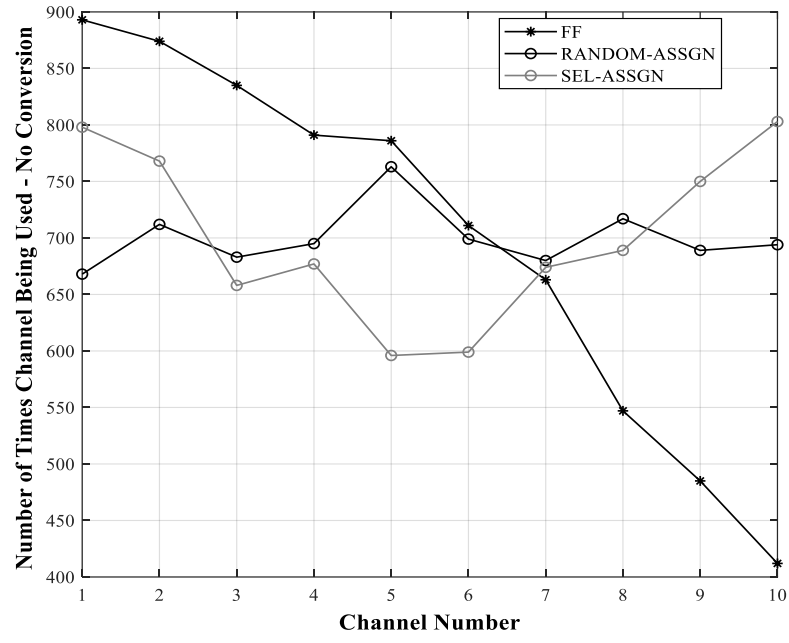

Fig.10. Frequency of channel usage by First Fit, Random Distribution and Selective Assignment methods for a load 8 Erlangs per link and with 15 Links (nodes), 10 Channels and 8000 iterations

An interesting feature of the proposed method can be observed in Fig.10, where the channels near the beginning of the sequences are reserved for the PUs and the channels near the end of the sequence are reserved for the SUs in case of selective assignment method. With this approach, the blocking probabilities can be significantly reduced in case of the selective assignment method. The feature of assigning different priority channels is not possible in case of first fit assignment and the random assignment method.

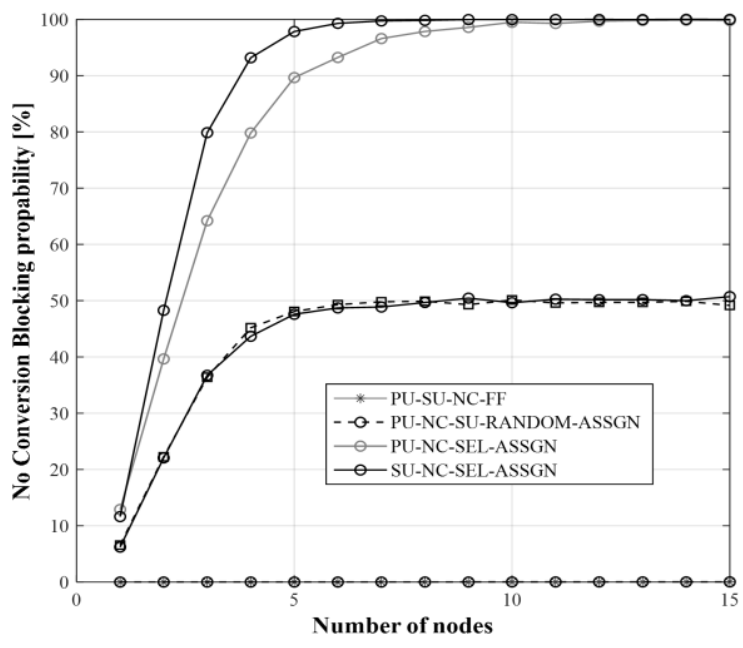

Fig.11. Blocking Probability of First Fit, Random Distribution and Selective Assignment methods for a load 8 Erlangs per link and with 15 Links (nodes), 10 Channels and 8000 iterations

In yet another simulation with the priority given near the start of the sequence for PU and the near the end of the sequence for SU, with equal probabilities of arrival of PU and SUs, the Fig.11 shows that there is significant improvement in the blocking probability for the selective assignment method. The reason behind the significant improvement is in case of first fit and the random assignment methods, the same set of channels are 
repeated channels both for PU and SU. With selective assignment method, the PU and SU can be reserved with a set of channels and hence there is significant reduction in the blocking probability. The blocking probability is maximum when the reserved channels in the selective channels are as distant apart as possible between PUs and SUs.

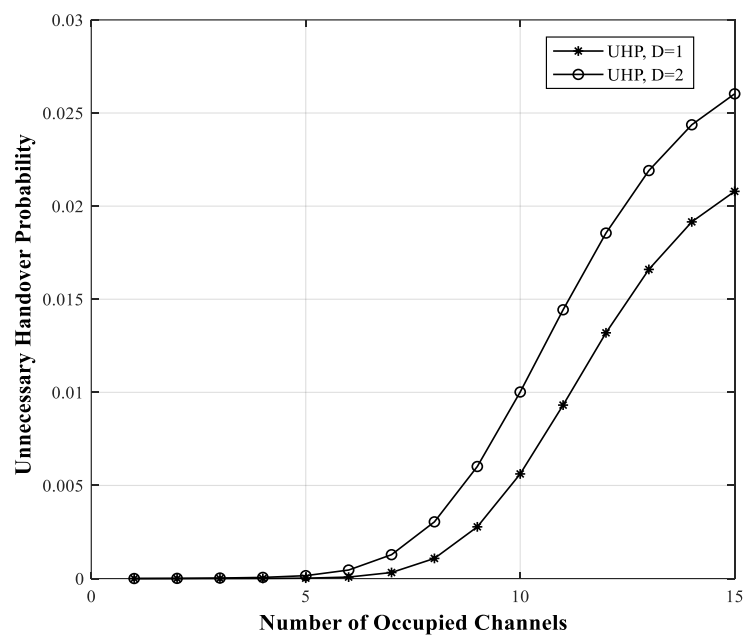

Fig.12. Unnecessary Handover Probability vs. number of occupied channels for $B_{1}=15, B_{2}=15$ and $B_{3}=15$.

From Fig.12, the UHP for different decision times can be read and it can be used along with the blocking probabilities of Fig.10 to determine the unsuccessful probability of handover of first fit, random assignment and selective assignment methods.

\section{CONCLUSIONS}

In this work, the channel assignment algorithms are implemented in MATLAB. The summary of results is presented in Table.1. The first fit method and random assignment method are used to estimate the blocking probabilities. The First fit method yielded lower blocking probabilities than the random assignment method, however random assignment method has the advantage of the utilizing all the channels randomly and efficiently. The advantages of the first fit method and random assignment are combined and a new assignment method, namely selective assignment is proposed. In this method, a window for selective channels for maximum assignment is randomly selected. The window can be selected anywhere in the sequence of the channels. Also when this assignment is applied to PU and SU together, the first fit and random and selective assignment methods will repeatedly use the same channels or channels in the same window for assignment. As future work, total unsuccessful probabilities need to be estimated when a PU gets blocked in the window reserved for PU, it can be assigned in the window of SU so that blocking probabilities can further be reduced for PUs.

Hence the first fit method and selective assignment methods yielded almost same blocking probability. However, in selective assignment method, there is a flexibility to choose multiple windows separately for PU and SU. It is shown in the results that as the distance between the window of PU and window of SU increases, the blocking probabilities become much better than the first fit and random assignment. When the distance is maximum, the blocking probability drops by $50 \%$ in case of selective assignment method. Hence, the selective assignment proposed in this paper is more efficient than that of the first fit and the random assignment methods. The blocking probabilities are combined with the unnecessary handover probabilities to determine the unsuccessful handover probabilities of the first fit, random assignment and selective assignment methods.

Table.1. Summary of all Simulations

\begin{tabular}{|c|c|c|c|c|}
\hline $\begin{array}{c}\text { Number of } \\
\text { Channels }\end{array}$ & $\begin{array}{c}\text { Number of } \\
\text { Nodes }\end{array}$ & $\begin{array}{c}\text { Load in } \\
\text { Erlangs }\end{array}$ & Ref & $\begin{array}{c}\text { Best } \\
\text { Assignment } \\
\text { method }\end{array}$ \\
\hline 10 & 10 & 3 & Fig.2 & First Fit \\
\hline 11 & 20 & 5 & Fig.4 & First Fit \\
\hline 10 & 15 & 8 & Fig.6 & $\begin{array}{c}\text { Selective } \\
\text { Assignment } \\
\text { and First Fit }\end{array}$ \\
\hline 50 & 20 & 15 & Fig.8 & $\begin{array}{c}\text { Selective } \\
\text { Assignment }\end{array}$ \\
\hline 10 & 15 & 8 & Fig.11 & $\begin{array}{c}\text { Selective } \\
\text { Assignment }\end{array}$ \\
\hline
\end{tabular}

\section{REFERENCES}

[1] Ejaz Ahmed, Abdullah Gani, Saeid Abolfazli, Liu Jie Yao and Samee U. Khan, "Channel Assignment Algorithms in Cognitive Radio Networks: Taxonomy, Open Issues, and Challenges", IEEE Communications Surveys and Tutorials, Vol. 18, No. 1, pp. 795-823, 2016.

[2] Elias Z, Tragos, Sherali Zeadally, Alexandros G, Fragkiadakis and Vasilios A. Siris, "Spectrum Assignment in Cognitive Radio Networks: A Comprehensive Survey", IEEE Communications Survey and Tutorials, Vol. 15, No. 3, pp. 1108-1135, 2013.

[3] Muhammad Rehan Usman and Soo Young Shin, "Channel Allocation Schemes for Permanent User Channel Assignment in Wireless Cellular Networks", IETE Journal of Research, Vol. 62, No. 2, pp. 189-197, 2016.

[4] Muhammad Rehan Usman and Soo Young Shin, "Channel Blocking Analysis and Availability Prediction in Cognitive Radio Networks", Proceedings of IEEE Workshop on Computing, Networking and Communications, pp. 1-6, 2017.

[5] Murtaza Zafer and Eytan Modiano, "Blocking Probability and Channel Assignment in Wireless Networks", IEEE Transactions on Wireless Communications, Vol. 5, No. 4, pp. 233-245, 2006.

[6] Xiukui Li and S.A. Reza Zekavat, "Cognitive Radio Based Spectrum Sharing: Evaluating Channel Availability via Traffic Pattern Prediction", Journal of Communications and Networks, Vol. 11, No. 2, pp. 104-114, 2009.

[7] Berk Canberk, Ian F. Akyildiz and Sema Oktug, "Primary User Activity Modeling using First-Difference Filter Clustering and Correlation in Cognitive Radio Networks", IEEE/ACM Transactions on Networking, Vol. 19, No. 1, pp. 170-183, 2011.

[8] Rahul Bajpai and Aditya Trivedi, "Joint Power and Spectrum Allocation for Cooperative Cognitive Radio Network with End User Mobility", Proceedings of $11^{\text {th }}$ 
International Conference on Wireless and Optical Communications Networks, pp. 1-5, 2014.

[9] Xiukui Li and S.A. Reza Zekavat, "Traffic Pattern Prediction Based Spectrum Sharing for Cognitive Radios", Proceedings of International conference on Electrical and Electronic Engineering, pp. 1-5, 2009.

[10] X. Li and S.A. Reza Zekavat, "Spectrum Sharing Across Multiple Service Providers via Cognitive Radio Nodes", IET Communication, Vol. 4, No. 5, pp. 551-5661, 2010.

[11] R. Kaniezhil and C. Chandrasekar, "Multiple Service providers sharing Spectrum using Cognitive Radio", International Journal of Scientific and Engineering Research, Vol. 3, No. 3, pp. 1-7, 2012.

[12] H. Jiang and S. Rappaport, "Prioritized Channel Borrowing without Locking: A Channel Sharing Strategy for Cellular Communications", IEEE/ACM Transactions on Networking, Vol. 4, No. 2, pp. 163-172, 1996.

[13] J.C.I. Chuang, "Performance Issues and Algorithms for Dynamic Channel Assignment", IEEE Journal on Selected Areas in Communications, Vol. 11, No. 6, pp. 955-963, 1993.

[14] R. Mathar and J. Mattfeldt, "Channel Assignment in Cellular Radio Networks", IEEE Transactions on Vehicular Technology, Vol. 42, No. 4, pp. 647-656, 1993

[15] G. Cao and M. Singhal, "Distributed Fault-Tolerant Channel Allocation for Cellular Networks", IEEE Journal of Selected Areas in Communications, Vol. 18, No. 7, pp. 1326-1337, 2000.

[16] J. Yang, D. Manivannan and M. Singhal, "A Fault-Tolerant Dynamic Channel Allocation Scheme for Enhancing QoS in
Cellular Networks", Proceedings of IEEE $36^{\text {th }}$ International Conference on System Sciences, pp. 306-315, 2003.

[17] T.H. Lai, J. Jiang and T. Ma, "A Relaxed Mutual Exclusion Problem with Application to Channel Allocation in Mobile Cellular Networks", Proceedings of IEEE $20^{\text {th }}$ International Conference on Distributed Computing Systems, pp. $592-$ $599,2000$.

[18] X. Dong and T.H. Lai, "Distributed Dynamic Carrier Allocation in Mobile Cellular Networks: Search vs. Update", Proceedings of International Conference on Distributed Computing Systems, pp. 108-115, 1997.

[19] R. Prakash, N. Shivaratri and M. Singhal, "Distributed Dynamic Channel Allocation for Mobile Computing", Proceedings of $14^{\text {th }}$ International Symposium on Principles of Distributed Computing, pp. 47-56, 1995.

[20] Jian Chang Yang and D. Manivannan, "Performance Comparison of Two Channel Allocation Approaches: Channel Pre-allocation vs. Non-Pre-allocation", Proceedings of $3^{\text {rd }}$ International Conference on Wireless and Mobile Communications, pp. 191-195, 2007.

[21] C. Chi, X. Cai, R. Hao and F. Liu, "Modeling and Analysis of Handover Algorithms", Proceedings of IEEE Global Telecommunications Conference, pp. 4473-4477, 2007.

[22] S. Akhila and S. Kumar, "Analysis of Handover Algorithms based on Wrong Decision Probability Model”, International Journal of Wireless Networks and Communications, Vol. 2, No. 1, pp. 165-173, 2010.

[23] Suresh R. Halhalli, Subhash Kulkarni, K.S.R. Anjaneyulu and S. Akhila, "Probability Modeling of Multi Node Wireless Networks", International Journal of Computer Applications, Vol. 51, No. 1, pp. 22-27, 2012. 